\title{
Gene expression and prognosis of NOX family members in gastric cancer
}

This article was published in the following Dove Press journal:

OncoTargets and Therapy

\section{Xin You' \\ Mingzhe $\mathrm{Ma}^{2,3}$ \\ Guoxin $\mathrm{Hou}^{4}$ \\ Yumin $\mathrm{Hu}^{2}$ \\ Xi Shi'}

'The First Department of Chemotherapy, The First Affiliated Hospital of Fujian Medical University, Fuzhou, China; ${ }^{2}$ State Key Laboratory of Oncology in South China, Sun Yat-Sen University Cancer Center, Collaborative Innovation Center for Cancer Medicine, Guangzhou, China; ${ }^{3}$ Department of Gastric Surgery, Sun Yat-Sen University Cancer Center, Guangzhou, China; ${ }^{4}$ Department of Oncology, The First Affiliated Hospital of Jiaxing University, Jiaxing, China
Correspondence: Xi Shi

The First Department of Chemotherapy, The First Affiliated Hospital of Fujian Medical University, 20 Cha Zhong Road, Fuzhou 350005, China

Tel +86 59l $8798 \quad 1708$

Fax +86 59| 87982836

Email shixiarticle@।63.com
Introduction: Nicotinamide adenine dinucleotide phosphate (NADPH) oxidases (NOX) are frequently deregulated in several human malignancies, including gastric cancer(GC). NOX-derived reactive oxygen species have been reported to contribute to gastric carcinogenesis and cancer progression. However, the expression and prognostic role of individual NOX in GC patients remain elusive. Methods and materials: We investigated genetic alteration and mRNA expression of NOX family in GC patients via the cBioPortal, Human Protein Atlas, and Oncomine databases. Furthermore, we evaluated prognostic value of distinct NOX in GC patients through "The Kaplan-Meier plotter" database.

Results: Our analysis demonstrated that mRNA deregulation of NOX genes was common alteration in GC patients. Compared with normal tissues, NOX1/2/4 mRNA expression levels in GC tissues were higher, while NOX5 and DUOX1/2 expression levels were lower. Importantly, our results indicated that high mRNA expression of NOX2 was associated with better overall survival whereas NOX4 and DUOX1 were correlated with worse overall survival in all GC patients, particularly in intestinal-type GC patients. In addition, our data also shed light on the diverse roles of individual NOX members in GC patients with different clinicopathological features, including human epidermal growth factor receptor 2 status, clinical stages, pathological grades, and different choices of treatments of GC patients.

Conclusion: These findings suggest that individual NOX family genes, especially NOX2/4, and DUOX1, are potential prognostic markers in GC and implicate that the use of NOX inhibitor targeting NOX4 and DUOX1 may be an effective strategy for GC therapy.

Keywords: NADPH oxidases, stomach cancer, online database, prognosis, drug target

\section{Introduction}

Gastric cancer (GC) is one of the leading causes of cancer-related deaths worldwide. Over 950,000 new GC cases are diagnosed each year. ${ }^{1}$ Although the progresses in early diagnosis and multimodal therapeutic modalities have improved overall survival (OS), prognosis of advanced GC patients remains poor with a median OS $<1$ year. ${ }^{2,3}$ Therefore, the development of prognostic biomarkers and drug targets is required and will provide better prognosis and more effective personalized treatments for GC patients.

The mammalian nicotinamide adenine dinucleotide phosphate (NADPH) oxidases (NOX), the major producers of reactive oxygen species (ROS), are composed of 7 unique members (NOX1-5 and DUOX1-2). NOX-derived ROS participates in many processes for which required by living cells and organisms, such as host immune defense, wound healing, and cell proliferation and differentiation. ${ }^{4,5}$ Moreover, evidence accumulated in recent years indicated that aberrant level of NOX-derived ROS may play a critical role in tumorigenesis and tumor progression. ${ }^{6-8}$ Upregulation of NOX has been frequently observed in cancers and proposed to be targets in therapy of diverse 
types of solid tumors, such as prostate, colon, and lung cancer. ${ }^{9-11}$ Meanwhile, some studies have also demonstrated that downregulation of NOX genes resulted in enhanced oncogenic properties of cancer cells. ${ }^{12,13}$ As extensive efforts have been made to focus on the roles of NOX in diverse cancers, increasing evidence suggested that the expression and functional significance of NOX family members varies by both isoform specificity and tumor origin.

Some studies have shown that NOX are important players in stomach cancer and cancer-prone conditions, such as chronic inflammation induced by Helicobacter pylori. ${ }^{14-16}$ However, the expression and prognostic role of NOX in GC patients remain elusive. In the present study, we first comprehensively explored the expression and prognosis of individual NOX family members in GC patients by mining the online databases, which may facilitate the discovery of new biomarkers and development of more effective drug targets for treatment of GC.

\section{Materials and methods}

\section{Frequency of gene alteration analysis}

Alteration of NOX genes status in GC patients was obtained from the online cBioPortal for Cancer Genomics (http:// www.cbioportal.org/). ${ }^{17}$ The dataset "Stomach Adenocarcinoma (TCGA, Provisional)" was used. Genomic profiles, including mutations, putative copy-number alterations, and mRNA expressions (RNA Seq V2 RSEM with $z$-scores $= \pm 2$ ) were selected for querying NOX1, CYBB (NOX2), NOX3, NOX4, NOX5, DUOX1, and DUOX2. Results were shown as OncoPrint with the number and frequency of alteration on 7 NOX genes demonstrated in the webpage. Then, the data were exported and plotted using Graphpad Prism software.

\section{Gene expression profiles}

NOX family mRNA expressions in GC patients were analyzed by 2 online platforms: The Human Protein Atlas (HPA) (https://www.proteinatlas.org) and Oncomine (http://www. oncomine.org/).

\section{The HPA}

The HPA database provides abundant data of transcriptome and proteomes in specific human tissues and consists of Tissue Atlas, Cell Atlas, and Pathology Atlas. All quantitative transcriptomics data (RNA-Seq) in the Pathology Atlas were retrieved from the Cancer Genome Atlas (TCGA). ${ }^{18}$ With the purpose of analyzing the expression differences of NOX family genes in GC patients, the FPKM (Fragments Per Kilobase of exon per Million fragments mapped) values of individual gene (NOX1, CYBB (NOX2), NOX3, NOX4,
NOX5, DUOX1, and DUOX2) in GC patients were downloaded from the Pathology Atlas and the values between the fifth and ninety-fifth percentiles were performed using Graphpad Prism software.

\section{Oncomine}

Oncomine is a collection of cancer microarray databases with an integrated data-mining platform. ${ }^{19}$ Relative mRNA expression of NOX genes (NOX1, NOX2, NOX4, NOX5, DUOX1, and DUOX2) in GC tissues compared with that in normal tissues was analyzed by Oncomine. Cui, Cho, and DErrico gastric datasets were selected due to that they were established on mRNA levels and contain larger samples $(\mathrm{n}>50)$. In Cui dataset, $p$-value $=0.05$ and top $10 \%$ gene rank were selected as threshold. In Cho and DErrico datasets, thresholds for significance were 1.5-fold change, $p$-value $=0.05$, and top $10 \%$ gene rank. The tenth, fiftieth, and ninetieth percentile data of each NOX isoform in both GC and normal tissues were plotted.

\section{Survival analysis and hazard ratios estimation}

The prognostic significance in GC patients with different expression levels of NOX isoforms were performed through the Kaplan-Meier plotter (http://kmplot.com/analysis/). Currently, breast cancer, lung cancer, ovarian cancer, and GC databases have been generated. ${ }^{20-23}$ The GC database (http:// kmplot.com/analysis/index.php? $p=$ service \& cancer=gastric) was established using gene expression data and survival information of 876 GC patients downloaded from Gene Expression Omnibus with clinical data. ${ }^{23}$ In order to evaluate the prognostic value of a specific gene, the patient cases were split into 2 groups according to the median expression of the gene (high vs low expression). The OS of GC patients was analyzed by using a Kaplan-Meier survival plot. Briefly, 6 NOX genes (NOX1, NOX2, NOX4, NOX5, DUOX1, and DUOX2) were entered into the GC database and analyzed with setting different clinical parameters. Then, Kaplan-Meier survival plots with hazard ratio (HR), 95\% CI, and $\log$-rank $p$ were displayed on the webpage. $p$-value of $<0.05$ was considered to be statistically significant. We downloaded data as text and replotted.

\section{Results}

\section{Genetic alteration and mRNA expression of NOX family in GC}

We first checked the genetic alterations of 7 NOX genes in GC patients using cBioPortal database. As shown in Figure $1 \mathrm{~A},>40 \%$ of GC patients showed genetic aberrations 
A

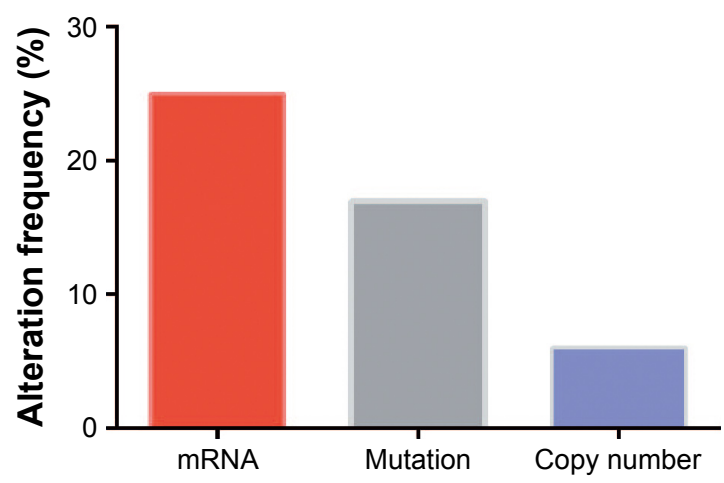

C

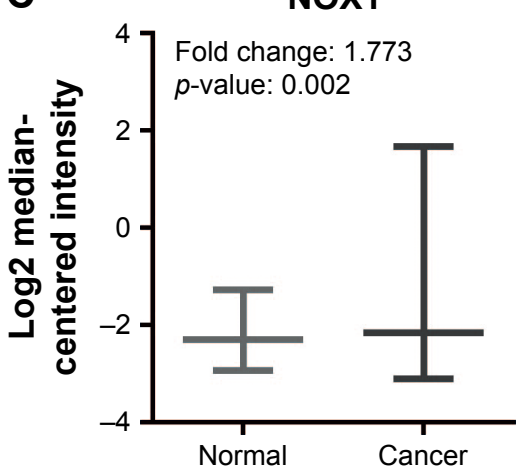

NOX5

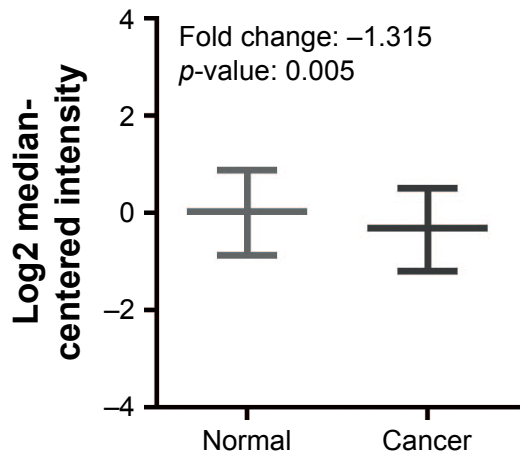

B

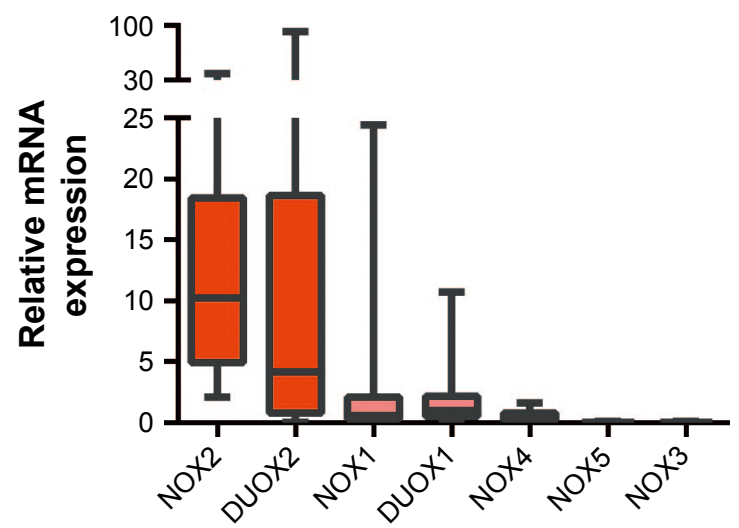

NOX2

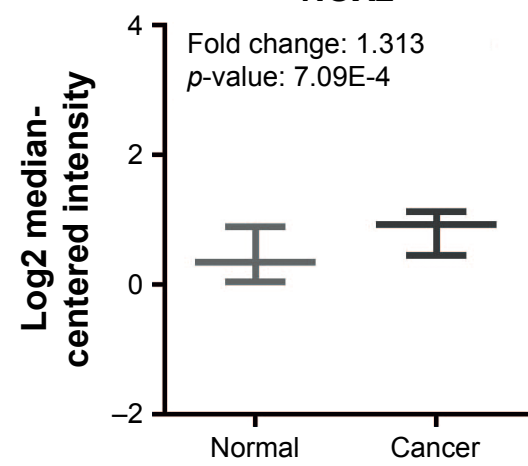

DUOX1

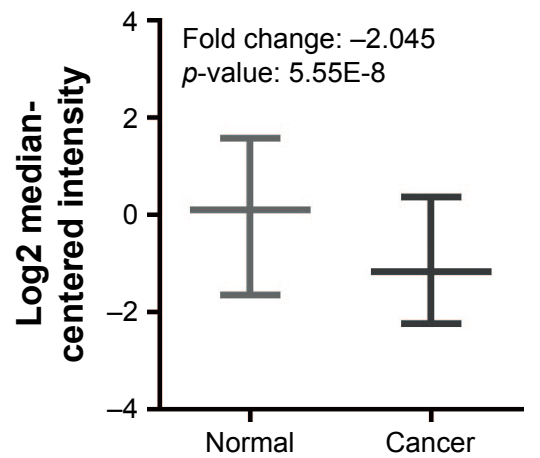

NOX4

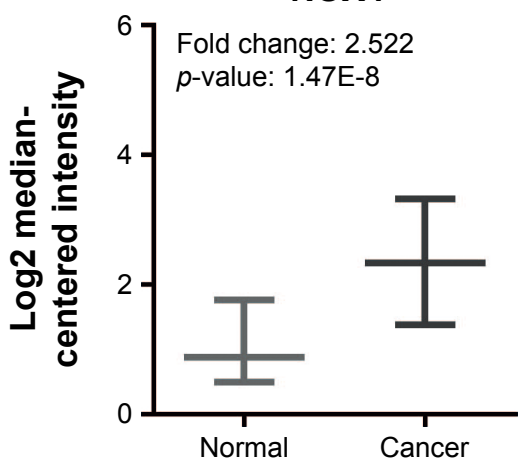

DUOX2

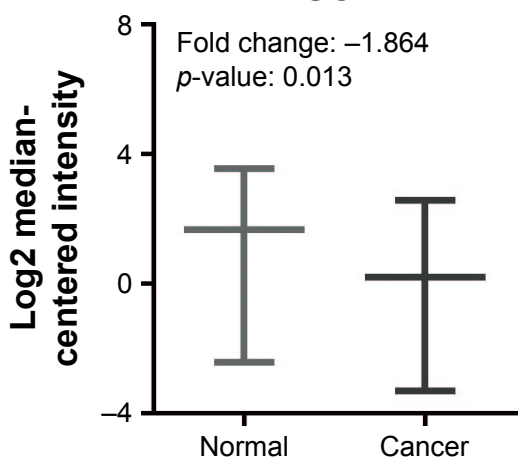

Figure I Genetic alteration and gene expression of NOX family in GC.

Notes: (A) Alteration frequency analysis of NOX genes in GC using cBioPortal. (B) Relative expression differences of NOX family genes in GC using Human Protein Atlas database. (C) Comparison of NOXI, NOX2, NOX4, NOX5, DUOXI, DUOX2 mRNA expression in gastric normal (left plot $n=80$ ) and cancer tissue (right plot $n=80$ ) in Cui dataset using Oncomine.

Abbreviations: GC, gastric cancer; NADPH, nicotinamide adenine dinucleotide phosphate; NOX, NADPH oxidases.

of NOX family genes, and the most common alteration was mRNA deregulation with alteration frequency of $25 \%$. Then we compared the mRNA level between different NOX family members in GC using HPA database, the NOX2 mRNA level was the highest among the NOX genes, whereas NOX5 and NOX3 expressions were very low (Figure 1B). Given that Salles et al had suggested that NOX5 mRNA was expressed in human stomach biopsies, ${ }^{24}$ we incorporated NOX5 into the following analysis. As for NOX3, its distribution has been reported to be highly limited in the inner ear and several fetal tissues; ${ }^{4,6,25}$ therefore, we excluded it from the following analysis and focused on the other 6 NOX genes mentioned in this article.

We also investigated relative mRNA expression of NOX genes in GC compared with that in normal tissue using Oncomine analysis. In Cui dataset, which contained the largest sample size $(n=160)$ on mRNA level among Oncomine gastric datasets, the mRNA levels of NOX1/2/4 in GC tissues were higher than in normal tissues, whereas NOX5 and DUOX1/2 expression levels were lower (Figure 1C). 
Table I Comparison of NOX genes expression in normal and different subtypes of GC tissues (Oncomine database, threshold by $p$-value 0.05 , fold change 1.5 , gene rank top $10 \%$ )

\begin{tabular}{|c|c|c|c|c|c|c|}
\hline Gene & $p$-value & $\begin{array}{l}\text { Fold } \\
\text { change }\end{array}$ & Dataset & $\begin{array}{l}\text { Normal } \\
\text { cases }\end{array}$ & $\begin{array}{l}\text { Cancer } \\
\text { cases }\end{array}$ & $\begin{array}{l}\text { Total } \\
\text { cases }\end{array}$ \\
\hline \multicolumn{7}{|c|}{ Intestinal type vs normal } \\
\hline \multirow[t]{2}{*}{ NOX4 } & $5.40 \mathrm{E}-4$ & 1.846 & Cho & 19 & 20 & 31 \\
\hline & $2.88 \mathrm{E}-8$ & 5.046 & DErrico & 31 & 26 & 57 \\
\hline DUOXI & I.4IE-6 & -3.618 & Cho & 19 & 20 & 39 \\
\hline \multirow[t]{2}{*}{ DUOX2 } & 0.002 & -1.723 & Cho & 19 & 20 & 39 \\
\hline & I.IIE-5 & -7.280 & DErrico & 31 & 26 & 57 \\
\hline \multicolumn{7}{|c|}{ Diffuse type vs normal } \\
\hline NOX2 & 0.004 & 1.906 & DErrico & 31 & 6 & 37 \\
\hline NOX4 & I.47E-8 & 2.522 & Cho & 19 & 31 & 50 \\
\hline DUOXI & $7.95 \mathrm{E}-7$ & -3.723 & Cho & 19 & 31 & 50 \\
\hline \multirow[t]{2}{*}{ DUOX2 } & 0.004 & -1.660 & Cho & 19 & 31 & 50 \\
\hline & 0.024 & -6.722 & DErrico & 31 & 6 & 37 \\
\hline \multicolumn{7}{|c|}{ Mixed type vs normal } \\
\hline NOX4 & 4.7IE-4 & 6.968 & DErrico & 31 & 4 & 35 \\
\hline NOX5 & 0.015 & -1.693 & DErrico & 31 & 4 & 35 \\
\hline \multirow[t]{2}{*}{ DUOXI } & I.44E-4 & -3.253 & Cho & 19 & 10 & 29 \\
\hline & 0.015 & -3.963 & DErrico & 31 & 4 & 35 \\
\hline
\end{tabular}

Abbreviations: GC, gastric cancer; NADPH, nicotinamide adenine dinucleotide phosphate; NOX, NADPH oxidases.

We further explored mRNA expression in Cho and DErrico gastric datasets, which separated GC cases into intestinal, diffuse, and mixed types. Relative change of distinct NOX members in different subtypes of GC are summarized in Table 1; it is notable that NOX4 and DUOX1 expressions were markedly higher and lower in all 3 types of GC compared with that in the normal controls.

\section{Prognostic values of NOX members in all GC patients}

Using Kaplan-Meier plotter analysis, we initially assessed the prognostic significance of the NOX isoforms in all GC patients. The survival curves are demonstrated in Figure 2. Among the 6 NOX genes we analyzed, NOX2 mRNA high expression was correlated with significantly better OS for all GC patients (HR 0.56 [95\% CI: 0.47-0.67], $p=3 \mathrm{E}-11$; Figure 2A). However, high NOX4 and DUOX1 expressions were significantly associated with a poor prognosis (NOX4, HR 1.47 [95\% CI: 1.18-1.82], $p=0.00049$; DUOX1, HR 1.45 [95\% CI: 1.22-1.72], $p=1.9 \mathrm{E}-05$; Figure $2 \mathrm{~B}$ and C). In addition, the mRNA expression levels of NOX1, NOX5, and DUOX2 were not correlated with OS (Figure 2D-F).

\section{Prognostic values of NOX members in different $\mathrm{GC}$ subtypes}

Histologically, GCs can be mostly diffuse or intestinal type according to the Lauren classification. ${ }^{1}$ Next, we examined the prognostic values of NOX members in the 2 main histological types. Similar to that in all GC patients, high mRNA expression of NOX2 showed favorable OS for intestinal-type GC patients (HR 0.59 [95\% CI: 0.43-0.81],
A

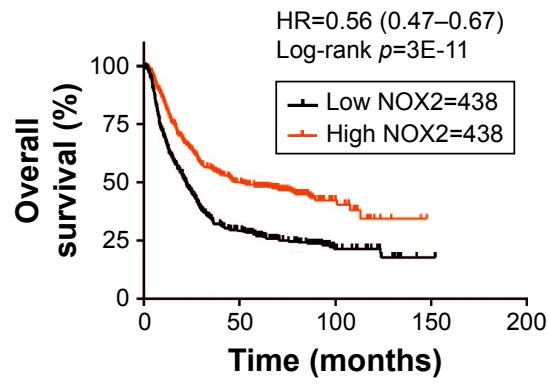

D

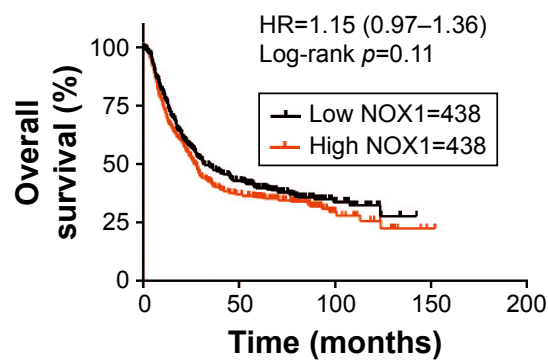

B

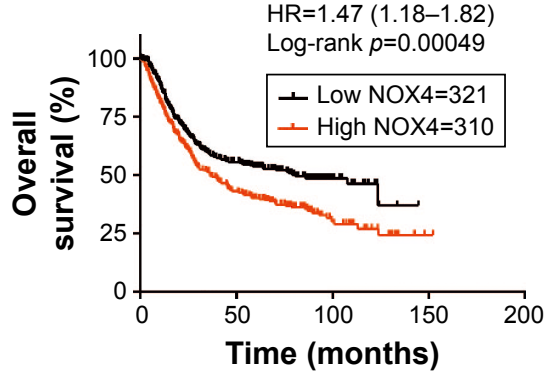

E

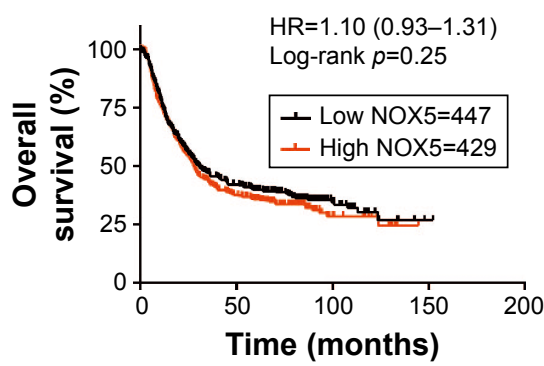

C Duox1

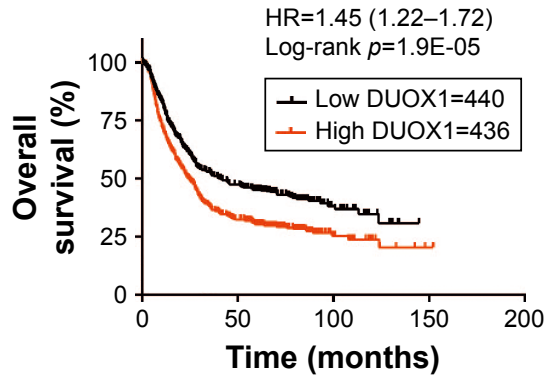

DUOX2

F

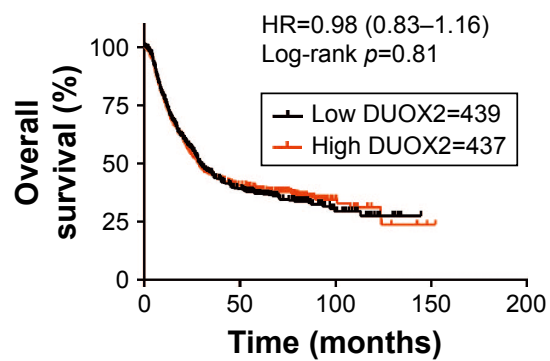

Figure 2 Survival analysis of NOX family genes in all GC patients using Kaplan-Meier plotter.

Notes: Survival curves of (A) NOX2 (Affymetrix IDs: 203923_s_at; n=876); (B) NOX4 (Affymetrix IDs: 236843_at; n=63I); (C) DUOXI (Affymetrix IDs: 219597_s_at; n=876); (D) NOXI (Affymetrix IDs: 2072I7_s_at; n=876); (E) NOX5 (Affymetrix IDs: 22064I_at; n=876); and (F) DUOX2 (Affymetrix IDs: 2 I9727_at; n=876).

Abbreviations: GC, gastric cancer; HR, hazard ratio; NADPH, nicotinamide adenine dinucleotide phosphate; NOX, NADPH oxidases. 
A

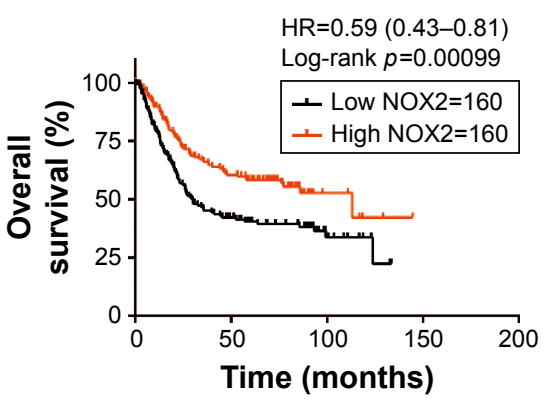

D

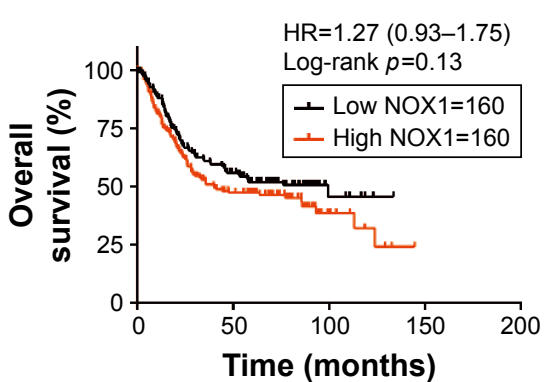

B

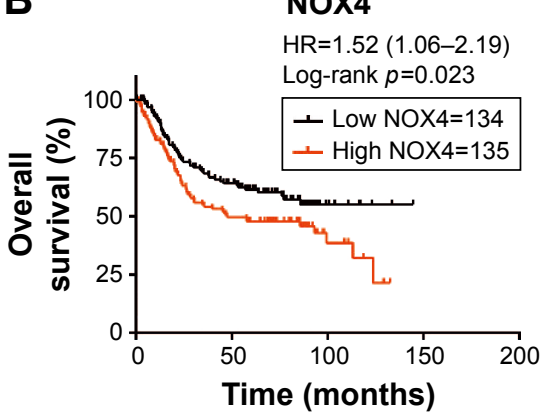

$\mathbf{E}$

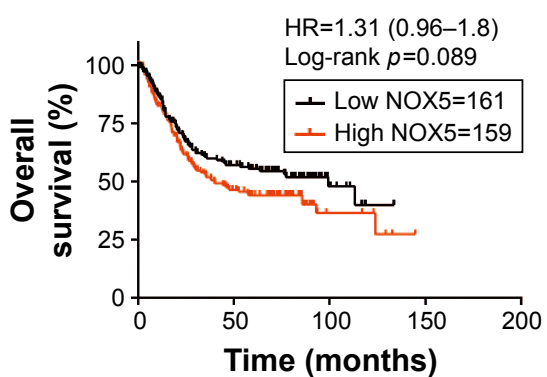

C

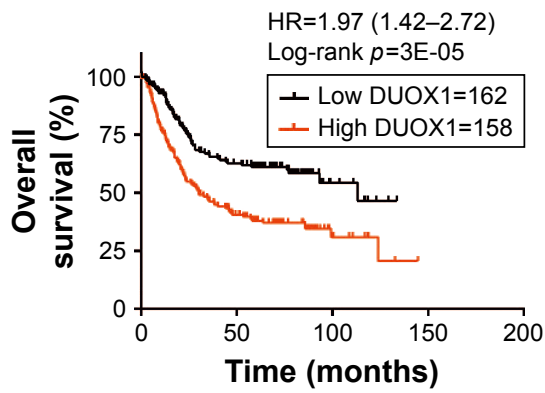

$\mathbf{F}$

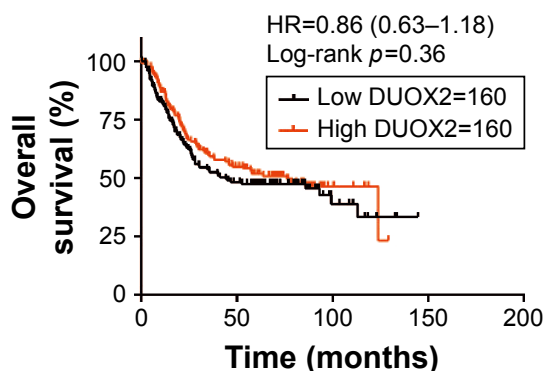

Figure 3 Survival analysis of NOX family genes in intestinal type GC patients using Kaplan-Meier plotter.

Notes: Survival curves of (A) NOX2 ( $n=320)$; (B) NOX4 ( $n=269)$; (C) DUOXI ( $n=320)$; (D) NOXI ( $n=320)$; (E) NOX5 ( $n=320)$; and (F) DUOX2 ( $n=320)$.

Abbreviations: GC, gastric cancer; HR, hazard ratio; NADPH, nicotinamide adenine dinucleotide phosphate; NOX, NADPH oxidases.

$p=0.00099$; Figure $3 \mathrm{~A}$ ), whereas high expressions of NOX4 and DUOX1 were significantly related with unfavorable prognosis in intestinal-type GC patients (NOX4, HR 1.52 [95\% CI: 1.06-2.19], $p=0.023$; DUOX1, HR 1.97 [95\% CI: $1.42-2.72], p=3 \mathrm{E}-05$; Figure $3 \mathrm{~B}$ and C). The mRNA expressions of NOX1, NOX5, and DUOX2 were not correlated with OS in intestinal-type GC (Figure 3D-F).

In patients with diffuse-type GC, only 3 members were found to be modestly associated with prognosis but without statistical difference. High NOX2 and DUOX2 expressions were modestly correlated with better OS (NOX, HR 0.75 [95\% CI: 0.53-1.05], $p=0.094$; DUOX2, HR 0.72 [95\% CI: 0.51-1.02], $p=0.06$; Figure 4A and B), and NOX4 was modestly correlated with worse OS (HR 1.35 [95\% CI: 0.96-1.91], $p=0.081$; Figure 4C). NOX1, NOX5, and DUOX1 expression were not related with prognosis in diffuse-type GC (Figure 4D-F).

\section{Prognostic values of NOX members in GC patients with different clinicopathological features}

Furthermore, we checked the relationship between NOX isoforms and other clinicopathological features for GC patients, such as human epidermal growth factor receptor 2 (HER2) status, clinical stages, pathological grades, and different choices of treatments. As from Table 2, high mRNA expression of NOX2 was associated with significantly better OS while the NOX4 and DUOX1 were correlated with worse OS in HER2-negative GC. Nevertheless, only DUOX1 mRNA high expression was associated with worse OS in HER2-positive GC patients. From Table 3, NOX2 high expression was associated with better OS and DUOX1 high expression was correlated with worse OS in both stage I/II and stage III/IV GC. In addition, elevated mRNA expressions of NOX4 and NOX5 were also found to be related with an unfavorable OS in stage III/IV GC patients. From Table 4, high expressions of DUOX2 and NOX4 indicated significantly worse OS in grades II and III GC, respectively. From Table 5, NOX1, NOX2, and NOX4 were significantly associated with different choice of treatment.

\section{Discussion}

ROS are oxygen-derived small molecules, including oxygen radicals (eg, superoxide anions $\left[\mathrm{O}_{2}^{-}\right]$, and nitric oxide [NO]) and certain nonradicals (eg, hydrogen peroxide $\left[\mathrm{H}_{2} \mathrm{O}_{2}\right]$, and hypochlorous acid [HOCl] $){ }^{4}$ The main product of NOX1, NOX2, NOX3, and NOX5 is superoxide anions, whereas the major detected product of NOX4 is $\mathrm{H}_{2} \mathrm{O}_{2}$, a more stable and membrane-permeable molecule. ${ }^{26}$ The generation of superoxide anions or $\mathrm{H}_{2} \mathrm{O}_{2}$ occurs either in the extracellular space or the intraorganellar space, such as endosomes and the endoplasmic reticulum. ${ }^{4,27}$ DUOX1 and DUOX2 primarily 


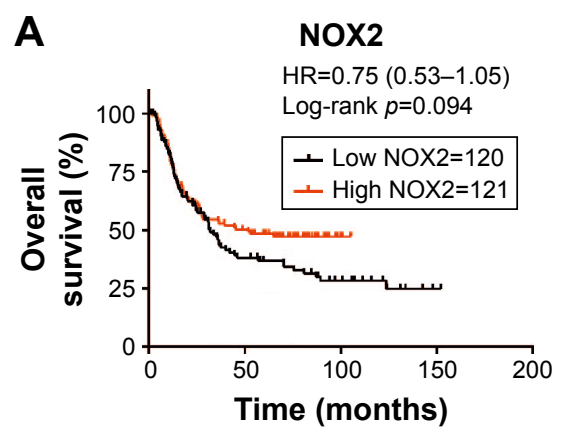

D

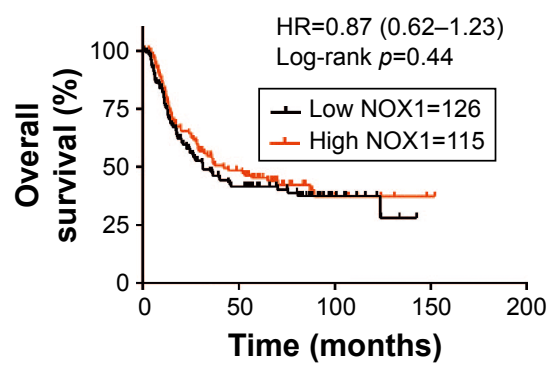

B

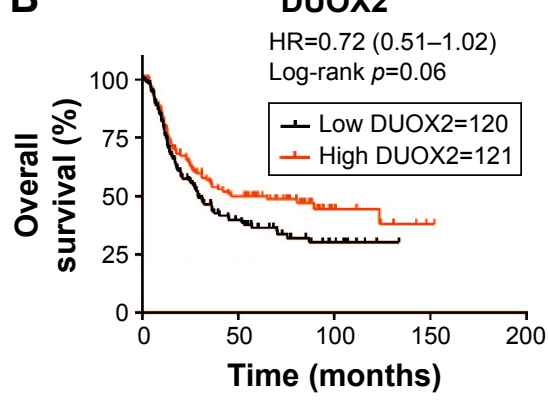

E

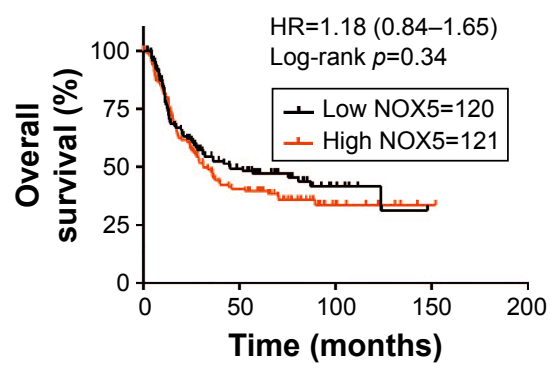

C

NOX4

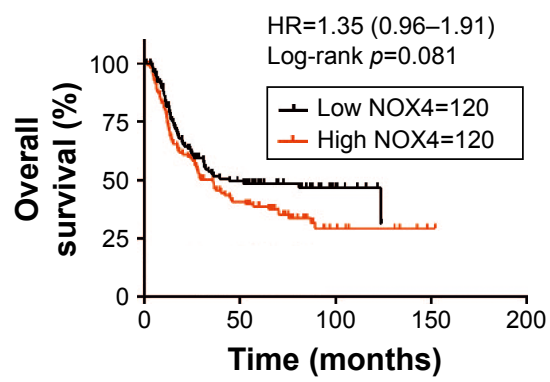

F Duox1

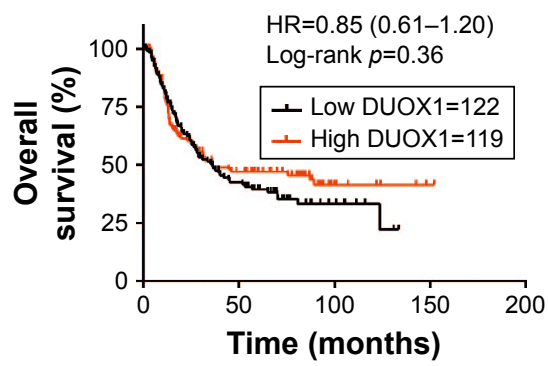

Figure 4 Survival analysis of NOX family genes in diffuse-type GC patients using Kaplan-Meier plotter.

Notes: Survival curves of (A) NOX2 ( $n=24 I)$; (B) DUOX2 ( $n=24 I)$; (C) NOX4 ( $n=240) ;(D)$ NOXI ( $n=24 I)$; (E) NOX5 ( $=24 I)$; and (F) DUOXI ( $n=24 I)$.

Abbreviations: GC, gastric cancer; HR, hazard ratio; NADPH, nicotinamide adenine dinucleotide phosphate; NOX, NADPH oxidases.

generate $\mathrm{H}_{2} \mathrm{O}_{2} \cdot{ }^{26}$ DUOX proteins are located at the apical membrane of the polarized epithelium, where they produce $\mathrm{H}_{2} \mathrm{O}_{2}$ in the extracellular space. ${ }^{27,28}$ Increasing evidence suggest that the superoxide anions and $\mathrm{H}_{2} \mathrm{O}_{2}$ generated by NOX isoforms are not only involved in physiological and pathophysiological processes that induce oncogenic effects, but also exhibit antitumor effect by ROS-dependent intercellular induction of apoptosis, a process that selectively eliminates malignant cells based on the $\mathrm{HOCl}$ and the $\mathrm{NO} /$ peroxynitrite-mediated signaling pathways. ${ }^{29}$ In order to

Table 2 Correlation of NOX members mRNA expression with HER2 status of GC patients

\begin{tabular}{lllll}
\hline Gene & HER2 & Cases & HR $(95 \%$ Cl) & p-value \\
& status & & & \\
\hline NOXI & Negative & 532 & $0.97(0.77-1.21)$ & 0.79 \\
& Positive & 344 & $1.03(0.80-1.34)$ & 0.81 \\
NOX2 & Negative & 532 & $0.48(0.38-0.60)$ & $<0.000 I^{*}$ \\
& Positive & 344 & $0.80(0.62-1.04)$ & 0.095 \\
NOX4 & Negative & 429 & $1.46(1.12-1.91)$ & $0.0048^{*}$ \\
& Positive & 202 & $1.34(0.92-1.95)$ & 0.12 \\
NOX5 & Negative & 532 & $1.04(0.84-1.31)$ & 0.70 \\
& Positive & 344 & $1.23(0.95-1.60)$ & 0.11 \\
DUOX1 & Negative & 532 & $1.28(1.02-1.60)$ & $0.033^{*}$ \\
& Positive & 344 & $1.30(1.00-1.68)$ & $0.049 *$ \\
DUOX2 & Negative & 532 & $0.88(0.71-1.11)$ & 0.28 \\
& Positive & 344 & $0.95(0.73-1.23)$ & 0.70 \\
\hline
\end{tabular}

Note: $*_{p}<0.05$.

Abbreviations: GC, gastric cancer; HR, hazard ratio; NADPH, nicotinamide adenine dinucleotide phosphate; NOX, NADPH oxidases. better understand the role of NOX family in GC, the present study explored the expression level and prognostic function of distinct NOX members in GC patients.

NOX1 has been reported to be overexpressed in various human solid tumors, including colon cancer, prostate cancer, and melanoma. ${ }^{5,30}$ Earlier studies indicated that NOX1 transcript was activated by $H$. pylori lipopolysaccharide in guinea pig gastric mucosal cells, ${ }^{31}$ and significantly expressed in human $\mathrm{H}$. pylori-associated gastritis and human. ${ }^{32}$ Moreover, elevated mRNA and protein levels of NOX1 were

Table 3 Correlation of NOX members mRNA expression with clinical stages of GC patients

\begin{tabular}{|c|c|c|c|c|}
\hline Gene & Stages & Cases & HR (95\% Cl) & $p$-value \\
\hline \multirow[t]{2}{*}{ NOXI } & $\mathrm{I}+\mathrm{II}$ & 207 & $\mathrm{I} .39(0.84-2.3 \mathrm{I})$ & 0.81 \\
\hline & III+IV & 453 & $1.09(0.87-1.31)$ & 0.47 \\
\hline \multirow[t]{2}{*}{ NOX2 } & $I+I I$ & 207 & $0.47(0.28-0.78)$ & $0.0098^{*}$ \\
\hline & III+IV & 453 & $0.62(0.48-0.77)$ & $<0.000$ I* \\
\hline \multirow[t]{2}{*}{ NOX4 } & $1+I I$ & 199 & I.II (0.64-I.90) & 0.9649 \\
\hline & III+IV & 337 & $1.42(1.09-1.87)$ & $0.016 *$ \\
\hline \multirow[t]{2}{*}{ NOX5 } & I+II & 207 & $0.9 \mid(0.55-|.5|)$ & 0.9095 \\
\hline & III+IV & 453 & $1.52(1.24-1.86)$ & $<0.000 I^{*}$ \\
\hline \multirow[t]{2}{*}{ DUOXI } & $\mathrm{I}+\mathrm{II}$ & 207 & $1.77(1.07-2.95)$ & $0.0044 *$ \\
\hline & III+IV & 453 & $1.37(1.03-1.82)$ & $0.0317 *$ \\
\hline \multirow[t]{2}{*}{ DUOX2 } & $\mathrm{I}+\mathrm{II}$ & 207 & $0.75(0.45-1.25)$ & 0.2332 \\
\hline & III+IV & 453 & $0.8 \mathrm{I}(0.64-1.0 \mathrm{I})$ & 0.0621 \\
\hline
\end{tabular}

Note: $* p<0.05$.

Abbreviations: GC, gastric cancer; HR, hazard ratio; NADPH, nicotinamide adenine dinucleotide phosphate; NOX, NADPH oxidases. 
Table 4 Correlation of NOX members mRNA expression with tumor grades of GC patients

\begin{tabular}{|c|c|c|c|c|}
\hline Gene & Grades & Cases & HR $(95 \% \mathrm{CI})$ & $p$-value \\
\hline \multirow[t]{3}{*}{ NOXI } & I & 32 & $0.53(0.22-1.27)$ & 0.15 \\
\hline & II & 67 & $0.80(0.42-1.55)$ & 0.51 \\
\hline & III & 165 & I.II (0.74-I.65) & 0.61 \\
\hline \multirow[t]{3}{*}{ NOX2 } & 1 & 32 & I.I3 (0.48-2.67) & 0.78 \\
\hline & II & 67 & $0.95(0.49-1.82)$ & 0.87 \\
\hline & III & 165 & $0.84(0.56-1.24)$ & 0.37 \\
\hline \multirow[t]{3}{*}{ NOX4 } & 1 & 5 & N/A & N/A \\
\hline & II & 67 & I.87 (0.97-3.6I) & 0.058 \\
\hline & III & $12 \mid$ & $1.64(1.01-2.67)$ & $0.044^{*}$ \\
\hline \multirow[t]{3}{*}{ NOX5 } & 1 & 32 & I.II (0.49-2.72) & 0.75 \\
\hline & ॥ & 67 & $0.78(0.4 \mid-1.50)$ & 0.46 \\
\hline & III & 165 & I.I6 (0.78-I.74) & 0.45 \\
\hline \multirow[t]{3}{*}{ DUOXI } & 1 & 32 & $2.14(0.88-5.21)$ & 0.085 \\
\hline & ॥ & 67 & I. $17(0.6 \mathrm{I}-2.23)$ & 0.64 \\
\hline & III & 165 & $0.92(0.62-1.37)$ & 0.68 \\
\hline \multirow[t]{3}{*}{ DUOX2 } & I & 32 & I.05 (0.44-2.47) & 0.91 \\
\hline & II & 67 & $1.99(1.03-3.86)$ & $0.037^{*}$ \\
\hline & III & 165 & $0.94(0.63-1.40)$ & 0.77 \\
\hline
\end{tabular}

Note: ${ }^{*} p<0.05$.

Abbreviations: GC, gastric cancer; HR, hazard ratio; N/A, not applicable; NADPH, nicotinamide adenine dinucleotide phosphate; NOX, NADPH oxidases.

observed in human GC, ${ }^{32,33}$ suggesting that NOX1 may be critical for inflammation-dependent carcinogenesis caused by $H$. pylori in human stomach. However, there is no report about the prognostic significance of NOX1 in GC patients. In this study, NOX1 expression was also found to be higher in GC tissues than in normal stomach tissues; nevertheless, mRNA level of NOX1 had no effect on OS of all GC patients, as well as intestinal- and diffuse-type cancer patients.

NOX2 was the first identified member of NOX family that has been recognized as an important player in malignant tumors. ${ }^{5}$ NOX2 was found to be a major source for ROS produced by vascular endothelial growth factor (VEGF)

Table 5 Correlation of NOX members mRNA expression with different treatment of GC patients

\begin{tabular}{lllll}
\hline Gene & Treatments & Cases & HR (95\% CI) & p-value \\
\hline NOXI & Surgery alone & 380 & $0.88(0.66-1.17)$ & 0.38 \\
& 5-FU-based adjuvant & 153 & $1.51(1.07-2.13)$ & $0.02^{*}$ \\
NOX2 & Surgery alone & 380 & $0.83(0.62-1.10)$ & 0.19 \\
& 5-FU-based adjuvant & 153 & $0.61(0.43-0.86)$ & $0.0042^{*}$ \\
NOX4 & Surgery alone & 380 & $1.47(1.10-1.97)$ & $0.0084^{*}$ \\
& 5-FU-based adjuvant & 34 & $1.63(0.66-4.07)$ & 0.29 \\
NOX5 & Surgery alone & 380 & $1.08(0.81-1.44)$ & 0.61 \\
& 5-FU-based adjuvant & 153 & $1.13(0.80-1.60)$ & 0.47 \\
DUOXI & Surgery alone & 38 & $1.10(0.83-1.47)$ & 0.50 \\
& 5-FU-based adjuvant & 153 & $0.89(0.63-1.26)$ & 0.52 \\
DUOX2 & Surgery alone & 380 & $0.92(0.69-1.22)$ & 0.55 \\
& 5-FU-based adjuvant & 153 & $1.21(0.86-1.71)$ & 0.27 \\
\hline
\end{tabular}

Note: $*_{p}<0.05$.

Abbreviations: GC, gastric cancer; HR, hazard ratio; NADPH, nicotinamide adenine dinucleotide phosphate; NOX, NADPH oxidases. and angiotensin 1 that involved in angiogenesis. Moreover, neovascularization in response to ischemia or VEGF was inhibited in NOX2-deficient mice. ${ }^{34} \mathrm{NOX} 2$ function in cancer development was also reported to be associated with ROSinduced immune suppression to cancer. ${ }^{8}$ Based on previous results, NOX2 seems to be a candidate oncogene. However, Zheng et al revealed that reduced NOX2 expression and activity mediated sleep fragmentation-induced accelerated tumor growth and invasiveness and indicated NOX2 may act as a tumor suppressor gene. ${ }^{13}$ Likewise, the role of NOX2 in GC was unclear and controversial. H. pylori-induced gastric inflammation as well as glandular atrophy, a precursor of intestinal-type GC, was found markedly increased in NOX2deficient mice. ${ }^{35}$ Nevertheless, Wang reported that NOX2 was upregulated in GC tissues and NOX2 protein-positive group of GC patients presented a poor prognosis compared with NOX2-negative groups. ${ }^{36}$ Intriguingly, our data analysis showed that overexpression of NOX2 mRNA in GC correlated with a better OS for all GC patients, particularly for intestinal-type GC. These paradoxical results may be due to different study design, specimens, detection way, and sample size. Moreover, it should be noted that, NOX2 is highly expressed in neutrophils and monocyte/macrophages. ${ }^{4}$ The mRNA expression level of NOX2 in GC tissues might be affected by blood contamination and/or the presence of phagocytes in the tumor tissues that use NOX2 for the generation of superoxide anions. Therefore, more efforts are highly needed to further elucidate the exact function and prognosis of NOX2 for malignancies, including GC.

The study about NOX3 in cancer is quite limited, predominantly attributed to its highly limited distribution in fetal tissues and inner ear. ${ }^{4,25}$ A study has linked NOX3 to development of hearing loss in patients receiving cisplatin treatment. ${ }^{37}$ Juhasz et al presented that NOX3 mRNA expression was almost absent in all tumor cell lines as well as the tumors and adjacent normal tissue samples they tested, including GC cell lines and stomach tissues. ${ }^{38}$ In line with these findings, our study showed that NOX3 mRNA level was the lowest among the NOX genes and undetectable in most of GC samples.

Among 7 NOX family members, NOX4 is relatively the most studied member in malignant tumors. Abundance of literature has showed that NOX4 was regarded as an oncoprotein in diverse types of solid tumors, such as colorectal cancer, breast cancer, prostate cancer, and glioblastoma. ${ }^{10,39-41}$ These researches also indicated the significant role of NOX4 in cancer proliferation, metastasis, anti-apoptosis, radiation resistance, and epithelial-to-mesenchymal transition. 
Recently, Gao et al revealed that NOX4 expression was elevated in GC tissues and involved in GC cell adhesion and invasion through Janus kinase/signal transducer and activator of transcription signaling pathways. ${ }^{16}$ Consistent with this result, our finding showed that NOX4 mRNA was significantly upregulated in GC tissues compared with normal tissues. High expression of NOX4 was correlated with worse OS in all GC patients, especially in intestinaltype GC patients. In addition, we observed that high mRNA expression of NOX4 indicated a worse OS in grade III and stage III/IV GC patients, but not in grade I/II and stage I/II GC patients, suggesting that NOX4 may contribute to the progression of GC and play an important role in late-stage and poorly differentiated GC.

NOX5-derived ROS is suggested to promote proliferation of prostate cancer cell by modulating the activity and expression of protein kinase C zeta and c-Jun N-terminal kinase, ${ }^{9}$ and enhance cell growth of melanoma through activation of hypoxia-inducible factor 1-alpha signaling pathways. ${ }^{42}$ But, so far, little is known about the function of NOX5 in GC. In the present study, we found NOX 5 mRNA expression was modestly lower in GC tissues compared with that in normal stomach tissues. NOX5 expression was not significantly correlated with $\mathrm{OS}$ in all GC patients. Interestingly, when we further explored the prognosis role of NOX5 in GC patients with other clinicopathological features, our data showed that the high expression of NOX5 mRNA indicated a worse OS in stage III/IV GC patients, but not in stage I/II GC patients. These observations implicated that NOX5 may be an unfavorable prognosis indicator for late-stage GC patients.

DUOXs (DUOX1 and DUOX2) contain an NADPH oxidase domain that generates $\mathrm{H}_{2} \mathrm{O}_{2}$ and a peroxidase domain that may exert the catalytic function. ${ }^{43,44}$ The expression and function of DUOXs vary across different cancers. While epigenetic silencing of DUOXs expression has been observed in lung cancer, ${ }^{45}$ upregulation of DUOXs was detected in colorectal cancer. ${ }^{15,38}$ High DUOXs expression was reported to be associated with reduced thyroid cancer mortality; ${ }^{46}$ whereas DUOXs-derived ROS was demonstrated to positively influence AKT signaling and promote cell survival in prostate cancer cells. ${ }^{47}$ Recently, Qi et al reported DUOX2 mRNA and protein levels in GC were increased compared with the adjacent nonmalignant tissues. ${ }^{15}$ However, in our study, DUOX1 and DUOX2 mRNA levels were both found to be decreased in GC tissues. The prognosis role of DUOXs in GC has, to our knowledge, not been studied. Paradoxically, our results showed that although DUOX1 was downregulated in GC tissues, low mRNA expression of DUOX1 was found to be correlated with better OS in all GC patients as well as intestinal-type cancer patients. In contrast to DUOX1, mRNA level of DUOX2 had no effect on OS of all GC patients. Low DUOX2 expression was modestly associated with worse $\mathrm{OS}$ in diffuse-type GC patients without statistical difference $(p=0.06)$. Further study is needed to confirm whether DUOX2 plays a function in diffuse-type GC.

HER2 is a member of the human epidermal growth factor receptor family that is responsible for regulating a variety of tumor biology. ${ }^{1}$ HER2 positivity in GC ranges from $6 \%$ to $34 \%$ of patients. $^{3}$ Targeted therapy with anti HER 2 antibody like Trastusumab has improved the OS in HER2-positive advanced GC patients. ${ }^{1,3}$ In this report, we found that high levels of NOX4 and DUOX1 were significantly associated with worse OS while NOX2 was associated with better OS in HER2-negative GC patients. In HER2-positive GC group, only DUOX1 mRNA high expression was found to be significantly correlated with the poor OS.

There is limited study on whether NOX expression status could impact the treatment efficiency of GC patients. Lee et al suggested NOX4 could serve as one of prognostic genes used to predict recurrence for GC patients after surgery. ${ }^{48}$ Consistent with this result, our study showed that an increased expression level of NOX4 was associated with unfavorable OS of GC patients who underwent surgery alone. Meanwhile, we found the high expression of NOX1 statistically showed unfavorable OS while NOX2 exhibited favorable OS for GC patients with 5-FU-based adjuvant therapy.

As NOX-derived ROS has been recognized as an important factor involved in cancer, NOX inhibitors are proposed as the promising therapeutic option. ${ }^{5,8}$ Historical NOX inhibitors, such as apocynin and diphenylene iodonium, are nonspecific and not isoform selective. ${ }^{8,49}$ Given the various roles that distinct NOX members play in different type of cancer, we expect highly specific inhibitors for the individual NOX members, like NOX4 or DUOX1 to be developed and validated for their usage in GC, especially in intestinal-type GC. Whether NOX isoforms, such as NOX4, are potential candidates for target inhibition in diffuse-type GC remain to be elucidated. In addition, the present study suggests that the usage of inhibitors targeting NOX2 in GC should be approached with caution.

\section{Conclusion}

By using online databases, we accessed the expression and prognosis of NOX members in GC patients. Importantly, high mRNA expression of NOX2 was found to be associated with better OS, whereas NOX4 and DUOX1 were correlated 
with worse OS in all GC patients. In addition, our data also shed light on the diverse roles played by individual NOX members in GC with different clinicopathological features. These findings suggest that individual NOX genes, especially NOX2/4 and DUOX1, are potential prognostic markers in GC and indicated that the use of NOX inhibitor targeting NOX4 and DUOX1 may be an effective strategy for GC therapy, but further exploration is needed.

\section{Acknowledgments}

We are grateful to the contributors of data to cBioPortal, HPA, Oncomine, and Kaplan-Meier plotter. This work was supported by Provincial Training Project of Young Talents (2015-ZQN-JC-24).

\section{Disclosure}

The authors report no conflicts of interest in this work.

\section{References}

1. Van Cutsem E, Sagaert X, Topal B, Haustermans K, Prenen H. Gastric cancer. Lancet. 2016;388(10060):2654-2664.

2. Yoon H, Kim N. Diagnosis and management of high risk group for gastric cancer. Gut Liver. 2015;9(1):5-17.

3. Jou E, Rajdev L. Current and emerging therapies in unresectable and recurrent gastric cancer. World J Gastroenterol. 2016;22(20):4812-4823.

4. Bedard K, Krause K. The NOX family of ROS-generating NADPH oxidases: physiology and pathophysiology. Physiol Rev. 2007;87(1): 245-313.

5. Block K, Gorin Y. Aiding and abetting roles of NOX oxidases in cellular transformation. Nat Rev Cancer. 2012;12(9):627-637.

6. Skonieczna M, Hejmo T, Poterala-Hejmo A, Cieslar-Pobuda A, Buldak RJ. NADPH oxidases: insights into selected functions and mechanisms of action in cancer and stem cells. Oxid Med Cell Longev. 2017;2017:1-15.

7. Bonner MY, Arbiser JL. Targeting NADPH oxidases for the treatment of cancer and inflammation. Cell Mol Life Sci. 2012;69(14):2435-2442.

8. Weyemi U, Redon C, Parekh P, Dupuy C, Bonner W. NADPH oxidases NOXs and DUOXs as putative targets for cancer therapy. Anticancer Agents Med Chem. 2013;13(3):502-514.

9. Holl M, Koziel R, Schafer G, et al. ROS signaling by NADPH oxidase 5 modulates the proliferation and survival of prostate carcinoma cells. Mol Carcinog. 2016;55(1):27-39.

10. Lin X, Yang L, Fu S, et al. Overexpression of NOX4 predicts poor prognosis and promotes tumor progression in human colorectal cancer. Oncotarget. 2017;8(20):33586-33600.

11. Han M, Zhang T, Yang L, Wang Z, Ruan J, Chang X. Association between NADPH oxidase (NOX) and lung cancer: a systematic review and meta-analysis. $J$ Thorac Dis. 2016;8(7):1704-1711.

12. Little A, Sham D, Hristova M, et al. DUOX1 silencing in lung cancer promotes EMT, cancer stem cell characteristics and invasive properties. Oncogenesis. 2016;5(10): e261.

13. Zheng J, Almendros I, Wang Y, et al. Reduced NADPH oxidase type 2 activity mediates sleep fragmentation-induced effects on TC1 tumors in mice. Oncoimmunology. 2015;4(2):e976057.

14. Tominaga K, Kawahara T, Sano T, et al. Evidence for cancer-associated expression of NADPH oxidase 1 (Nox1)-based oxidase system in the human stomach. Free Radic Biol Med. 2007;43(12):1627-1638.

15. Qi R, Zhou Y, Li X, et al. DUOX2 expression is increased in barrett esophagus and cancerous tissues of stomach and colon. Gastroenterol Res Pract. 2016;2016:1835684.
16. Gao X, Sun J, Huang C, Hu X, Jiang N, Lu C. RNAi-mediated silencing of NOX4 inhibited the invasion of gastric cancer cells through JAK2/ STAT3 signaling. Am J Transl Res. 2017;9(10):4440-4449.

17. Gao J, Aksoy B, Dogrusoz U, et al. Integrative analysis of complex cancer genomics and clinical profiles using the cBioPortal. Sci Signal. 2013;6(269):pl1.

18. Uhlen $\mathrm{M}$, Zhang $\mathrm{C}$, Lee $\mathrm{S}$, et al. A pathology atlas of the human cancer transcriptome. Science. 2017;357(6352):eaan2507.

19. Rhodes DR, Kalyana-Sundaram S, Mahavisno V, et al. Oncomine 3.0: genes, pathways, and networks in a collection of 18,000 cancer gene expression profiles. Neoplasia. 2007;9(2):166-180.

20. Györffy B, Lanczky A, Eklund A, et al. An online survival analysis tool to rapidly assess the effect of 22,277 genes on breast cancer prognosis using microarray data of 1,809 patients. Breast Cancer Res Treat. 2010; 123(3):725-731.

21. Győrffy B, Surowiak P, Budczies J, Lánczky A. Online survival analysis software to assess the prognostic value of biomarkers using transcriptomic data in non-small-cell lung cancer. PLoS One. 2013; 8(12):e82241.

22. Gyorffy B, Lánczky A, Szállási Z. Implementing an online tool for genome-wide validation of survival-associated biomarkers in ovariancancer using microarray data from 1287 patients. Endocr Relat Cancer. 2012;19(2):197-208.

23. Szász A, Lánczky A, Nagy Á, et al. Cross-validation of survival associated biomarkers in gastric cancer using transcriptomic data of 1,065 patients. Oncotarget. 2016;7(31):49322-49333.

24. Salles N, Szanto I, Herrmann F, et al. Expression of mRNA for ROSgenerating NADPH oxidases in the aging stomach. Exp Gerontol. 2005; 40(4):353-357.

25. Cheng G, Cao Z, Xu X, van Meir E, Lambeth J. Homologs of gp91phox: cloning and tissue expression of Nox3, Nox4, and Nox5. Gene. 2001; 269(1-2):131-140.

26. Lambeth JD, Neish AS. Nox enzymes and new thinking on reactive oxygen: a double-edged sword revisited. Аnпu Rev Pathol. 2014;9: $119-145$.

27. Leto TL, Morand S, Hurt D, Ueyama T. Targeting and regulation of reactive oxygen species generation by Nox family NADPH oxidases. Antioxid Redox Signal. 2009;11(10):2607-2619.

28. Little AC, Sulovari A, Danyal K, Heppner DE, Seward DJ, van der Vliet A. Paradoxical roles of dual oxidases in cancer biology. Free Radi Biol Med. 2017;110:117-132.

29. Riethmuller M, Burger N, Bauer G. Singlet oxygen treatment of tumor cells triggers extracellular singlet oxygen generation, catalase inactivation and reactivation of intercellular apoptosis-inducing signaling. Redox Biol. 2015;6:157-168.

30. Deep G, Kumar R, Jain AK, et al. Graviola inhibits hypoxia-induced NADPH oxidase activity in prostate cancer cells reducing their proliferation and clonogenicity. Sci Rep. 2016;6:23135.

31. Kawahara T, Kohjima M, Kuwano Y, et al. Helicobacter pylori lipopolysaccharide activates Rac1 and transcription of NADPH oxidase Nox1 and its organizer NOXO1 in guinea pig gastric mucosal cells. Am J Physiol Cell Physiol. 2005;288(2):C450-C457.

32. Augusto AC, Miguel F, Mendonca S, Pedrazzoli J Jr, Gurgueira SA. Oxidative stress expression status associated to Helicobacter pylori virulence in gastric diseases. Clin Biochem. 2007;40(9-10):615-622.

33. Montalvo-Jave EE, Olguin-Martinez M, Hernandez-Espinosa DR, et al. Role of NADPH oxidases in inducing a selective increase of oxidant stress and cyclin D1 and checkpoint 1 over-expression during progression to human gastric adenocarcinoma. Eur J Cancer. 2016;57: $50-57$.

34. Ushio-Fukai M, Nakamura Y. Reactive oxygen species and angiogenesis: NADPH oxidase as target for cancer therapy. Cancer Lett. 2008;266(1):37-52.

35. Keenan JI, Peterson RA 2nd, Hampton MB. NADPH oxidase involvement in the pathology of Helicobacter pylori infection. Free Rad Biol Med. 2005;38(9):1188-1196. 
36. Wang P. Relationship between expression of NADPH oxidase 2 and invasion and prognosis of human gastric cancer. World J Gastroenterol. 2015;21(20):6271.

37. Mukherjea D, Jajoo S, Kaur T, Sheehan KE, Ramkumar V, Rybak LP. Transtympanic administration of short interfering (si)RNA for the NOX3 isoform of NADPH oxidase protects against cisplatin-induced hearing loss in the rat. Antioxid Redox Signal. 2010;13(5):589-598.

38. Juhasz A, Ge Y, Markel S, et al. Expression of NADPH oxidase homologues and accessory genes in human cancer cell lines, tumours and adjacent normal tissues. Free Rad Res. 2009;43(6):523-532.

39. Boudreau H, Casterline B, Rada B, Korzeniowska A, Leto T. Nox4 involvement in TGF-beta and SMAD3-driven induction of the epithelial-to-mesenchymal transition and migration of breast epithelial cells. Free Radic Biol Med. 2012;53(7):1489-1499.

40. Kumar B, Koul S, Khandrika L, Meacham R, Koul H. Oxidative stress is inherent in prostate cancer cells and is required for aggressive phenotype. Cancer Res. 2008;68(6):1777-1785.

41. Hsieh C, Wu C, Lee H, Liang J, Yu C, Lin Y. NADPH oxidase subunit 4 mediates cycling hypoxia-promoted radiation resistance in glioblastoma multiforme. Free Radic Biol Med. 2012;53(4):649-658.

42. Antony $\mathrm{S}$, Jiang $\mathrm{G}, \mathrm{Wu} \mathrm{Y}$, et al. NADPH oxidase 5 (NOX5)-induced reactive oxygen signaling modulates normoxic HIF-1alpha and p27(Kip1) expression in malignant melanoma and other human tumors. Mol Carcinog. 2017;56(12):2643-2662.
43. van der Hoeven R, Cruz MR, Chavez V, Garsin DA. Localization of the dual oxidase BLI-3 and characterization of its NADPH oxidase domain during infection of Caenorhabditis elegans. PLoS One. 2015;10(4): e0124091.

44. Kim SH, Lee WJ. Role of DUOX in gut inflammation: lessons from Drosophila model of gut-microbiota interactions. Front Cell Infect Microbiol. 2014;3:116.

45. Luxen S, Belinsky SA, Knaus UG. Silencing of DUOX NADPH oxidases by promoter hypermethylation in lung cancer. Cancer Res. 2008; 68(4):1037-1045.

46. Pulcrano M, Boukheris H, Talbot M, et al. Poorly differentiated follicular thyroid carcinoma: prognostic factors and relevance of histological classification. Thyroid. 2007;17(7):639-646.

47. Pettigrew C, Clerkin J, Cotter T. DUOX enzyme activity promotes AKT signalling in prostate cancer cells. Anticancer Res. 2012;32(12): 5175-5181.

48. Lee J, Sohn I, Do I, et al. Nanostring-based multigene assay to predict recurrence for gastric cancer patients after surgery. PLoS One. 2014;9(3): e90133.

49. Altenhofer S, Radermacher KA, Kleikers PW, Wingler K, Schmidt HH. Evolution of NADPH oxidase inhibitors: selectivity and mechanisms for target engagement. Antioxid Redox Signal. 2015;23(5):406-427.
OncoTargets and Therapy

\section{Publish your work in this journal}

OncoTargets and Therapy is an international, peer-reviewed, open access journal focusing on the pathological basis of all cancers, potential targets for therapy and treatment protocols employed to improve the management of cancer patients. The journal also focuses on the impact of management programs and new therapeutic agents and protocols on

\section{Dovepress}

patient perspectives such as quality of life, adherence and satisfaction. The manuscript management system is completely online and includes a very quick and fair peer-review system, which is all easy to use. Visit http://www.dovepress.com/testimonials.php to read real quotes from published authors. 